\title{
ON SONINE'S INTEGRAL FORMULA AND ITS GENERALIZATION
}

\author{
HSIEN-YÜ HSÜ
}

1. Introduction. Let $J_{\nu}(x)$ denote Bessel function of order $\nu$ and let $\nu>-1 / 2$. The value of the integral

$$
S(\nu ; a, b, c)=\int_{0}^{\infty} J_{\nu}(a x) J_{\nu}(b x) J_{\nu}(c x) x^{1-\nu} d x,
$$

where $a, b, c$ are positive parameters, was evaluated by Sonine [8, p. 411 ${ }^{1}$ and given by his formula

$$
S(\nu ; a, b, c)=\frac{2^{\nu-1} \Delta^{2 \nu-1}}{\Gamma(1 / 2) \Gamma(\nu+1 / 2)(a b c)^{\nu}} \quad \text { or } 0,
$$

according as a triangle can, or cannot, be constructed with sides $a, b, c$. The area of the triangle is denoted by $\Delta$. Derivation of (1.2) may be established by means of a limiting process from either Dougall's integral formula or Dougall's expansion formula, both of which involve the product of three ultra-spherical polynomials [5, pp. 379382 ]. It has further been remarked [5, p. 375] that a proof of Sonine's theorem (1.2) can also be given by a procedure similar to that employed for the proof of the latter of Dougall's results by making use of Hankel's inversion formula.

In this paper, (a) we shall show the content of the previous remark with detailed explanations. (b) By use of the result (2.7) and Sonine's formula (1.2) a generalization of Sonine's integral (3.6) can be obtained. (c) Similarly, a generalization of Dougall's expansion formula may also be derived, which will contain (3.6) as a limiting case. And (d) we shall further give an explicit expression of the generalization of Dougall's integral formula, whence by means of a limiting process we also get the same generalized Sonine's integral. For $\nu=0$, the well known Nicholson result $[8$, p. 414$]$ can be displayed as a special case of (3.6).

2. Derivation of Sonine's integral (1.2) by means of Hankel's inversion formula. We shall assume that the area of the triangle as constructed by the given lengths $a, b, c$ is greater than zero. For the special case when the area $\Delta$ is zero, then we may replace the expres-

Received by the editors March 6, 1948.

1 Numbers in brackets refer to the references cited at the end of the paper. 
$\operatorname{sion} \Delta^{2 \nu-1}$ in the right member of (1.2) by $0,1 / 2$ and $+\infty$ for $\nu>1 / 2$, $\nu=1 / 2$ and $\nu<1 / 2$ respectively $[7$, p. 685]. When the construction of the triangle becomes impossible it is easy to show that the value of the integral (1.1) vanishes [8, p. 413].

Let us consider a plane triangle with two fixed sides $\beta$ and $\gamma$, and that the angle $\phi$ between $\beta$ and $\gamma$ should vary from 0 to $\pi$. Then the opposite side $\alpha$ to the angle $\phi$ of the triangle can be evaluated from the cosine law, namely:

$$
\alpha^{2}=\beta^{2}+\gamma^{2}-2 \beta \gamma \cos \phi .
$$

The addition theorem for Bessel function of order $\nu[8$, p. 363] gives

$$
\frac{J_{\nu}(\alpha)}{\alpha^{\nu}}=2^{\nu} \Gamma(\nu) \sum_{m=0}^{\infty}(\nu+m) \frac{J_{\nu+m}(\beta) J_{\nu+m}(\gamma)}{(\beta \gamma)^{\nu}} P_{m}^{(\nu)}(\cos \phi),
$$

which is valid for all values of $\beta, \gamma$ and $\phi$, and for all values of $\nu$ with the exception of ${ }^{2} 0,-1,-2, \cdots$. Hence we have by integration

$$
\begin{aligned}
\int_{0}^{\pi} \frac{J_{\nu}(\alpha)}{\alpha^{\nu}} \sin ^{2 \nu} \phi d \phi & =2^{\nu} \Gamma(\nu+1) \frac{J_{\nu}(\beta) J_{\nu}(\gamma)}{(\beta \gamma)^{\nu}} \int_{0}^{\pi} \sin ^{2 \nu} \phi d \phi \\
& =2^{\nu} \Gamma(\nu+1 / 2) \pi^{1 / 2} \frac{J_{\nu}(\beta) J_{\nu}(\gamma)}{(\beta \gamma)^{\nu}} .
\end{aligned}
$$

The above formula (2.3) holds true even when $\nu=0$ (see footnote 2). Now we introduce $\alpha$ instead of $\phi$ as the variable of the integration. Since

$$
\beta \gamma \sin \phi=2 \Delta_{1},
$$

where $\Delta_{1}$ is the area of the triangle with sides $\alpha, \beta, \gamma$; and since $\beta \gamma \sin \phi d \phi=\alpha d \alpha$, we find for the left member of (2.3)

$$
\int_{0}^{\pi} \frac{J_{\nu}(\alpha)}{\alpha^{\nu}} \sin ^{2 \nu-1} \phi \sin \phi d \phi=\frac{2^{2 \nu-1}}{(\beta \gamma)^{2 \nu}} \int_{|\beta-\gamma|}^{\beta+\gamma} \frac{J_{\nu}(\alpha)}{\alpha^{\nu}} \Delta_{1}^{2 \nu-1} \alpha d \alpha .
$$

After this transformation, (2.3) can be written in the form

$$
\int_{|\beta-\gamma|}^{\beta+\gamma} \frac{2^{\nu-1} J_{\nu}(\alpha) \Delta_{1}^{2 \nu-1}}{\Gamma(1 / 2) \Gamma(\nu+1 / 2)(\alpha \beta \gamma)^{\nu}} \alpha d \alpha=J_{\nu}(\beta) J_{\nu}(\gamma)
$$

Put $\alpha=R x, \beta=b x, \gamma=c x$, we obtain

\footnotetext{
${ }^{2}$ In case $\nu=0$, the addition theorem is given by $\left(2.2^{\prime}\right) J_{0}(\alpha)=\sum_{m-0}^{\infty} \epsilon_{m} J_{m}(\beta) J_{m}(\gamma)$ - $\cos m \phi$, where $\epsilon_{0}=1 ; \epsilon_{m}=2, m \geqq 1$. By integration, we have $\left(2.3^{\prime}\right)(1 / \pi) \int^{\pi} J_{0}(\alpha) d \phi$ $=J_{0}(\beta) J_{0}(\gamma)$, which is indeed the particular form of (2.3) when $\nu=0$.
} 


$$
\begin{aligned}
\int_{|b-c|}^{b+c} \frac{2^{\nu-1} J_{\nu}(R x) \Delta_{2}^{2 \nu-1}}{\Gamma(1 / 2) \Gamma(\nu+1 / 2)(R b c)^{\nu}} R d R & =\int_{0}^{\infty} f(R) J_{\nu}(R x) R d R \\
& =x^{-\nu} J_{\nu}(b x) J_{\nu}(c x),
\end{aligned}
$$

where $\Delta_{2}$ is the area of the triangle with sides $R, b, c$ and where we set (2.8) $f(R)=\left\{\begin{array}{cr}\frac{2^{\nu-1} \Delta_{2}^{2 \nu-1}}{\Gamma(1 / 2) \Gamma(\nu+1 / 2)(R b c)}, & |b-c| \leqq R \leqq b+c, \\ 0, & R<|b-c|, \text { or } R>b+c .\end{array}\right.$

Now the function $f(R)$ as defined above in (2.8) satisfies the condition that

$$
\int_{0}^{\infty} f(R) R^{1 / 2} d R
$$

exists and is absolutely convergent for $\nu>-1 / 2$. Therefore we can apply Hankel's inversion formula $[8$, p. 456] and have

$$
\begin{aligned}
S(\nu ; a, b, c) & =\int_{0}^{\infty} x J_{\nu}(a x)\left\{\int_{0}^{\infty} f(R) J_{\nu}(R x) R d R\right\} d x \\
& =\frac{1}{2}\{f(a-0)+f(a+0)\}=f(a),
\end{aligned}
$$

provided that the positive number " $a$ " lies inside the interval in which $f(R)$ is of bounded variation. This is actually satisfied because of the fact that $f(R)$ has a bounded derivative for the whole interval except the end points $R=|b-c|$ and $R=b+c$, at which points the area of the corresponding triangle vanishes.

3. Generalization of Sonine's integral formula. We are given four positive parameters $a, b, c, d$, say $a \geqq b \geqq c \geqq d$ and let $2 \sigma=a+b+c+d$. When a quadrilateral can not be constructed with lengths $a, b, c, d$ as sides, the value of the integral

$$
S(\nu ; a, b, c, d)=\int_{0}^{\infty} J_{\nu}(a x) J_{\nu}(b x) J_{\nu}(c x) J_{\nu}(d x) x^{1-2 \nu} d x
$$

may easily be shown to vanish $[8$, p. 413]. Moreover, from the well known asymptotic formula for Bessel functions [8, p. 199]

$$
J_{\nu}(x)=\left(\frac{2}{\pi x}\right)^{1 / 2} \cos \left(x-\frac{\nu \pi}{2}-\frac{\pi}{4}\right)+O\left(\frac{1}{x^{3 / 2}}\right), \quad x \rightarrow \infty,
$$


the principal part of (3.1) will become

$$
\begin{aligned}
\left(\frac{2}{\pi}\right)^{2} \frac{1}{(a b c d)^{1 / 2}} \int_{0}^{1} t^{-1-2 \nu}\left\{\cos \left(a t-\frac{\nu \pi}{2}-\frac{\pi}{4}\right)\right. \\
\\
\cos \left(b t-\frac{\nu \pi}{2}-\frac{\pi}{4}\right) \cos \left(c t-\frac{\nu \pi}{2}-\frac{\pi}{4}\right) \\
\left.\cos \left(d t-\frac{\nu \pi}{2}-\frac{\pi}{4}\right)\right\} d t .
\end{aligned}
$$

We can point out without difficulty the conditions under which the integral (3.1) diverges. Recalling the identity

$$
\begin{aligned}
8 \cos (m \phi & \left.-\frac{\nu \pi}{2}-\frac{\pi}{4}\right) \cos \left(n \phi-\frac{\nu \pi}{2}-\frac{\pi}{4}\right) \\
& \cdot \cos \left(p \phi-\frac{\nu \pi}{2}-\frac{\pi}{4}\right) \cos \left(q \phi-\frac{\nu \pi}{2}-\frac{\pi}{4}\right) \\
= & \cos \{(m+n+p+q) \phi-(2 \nu+1) \pi\} \\
& +\cos \left\{(n+p+q-m) \phi-\nu \pi-\frac{\pi}{2}\right\} \\
& +\cos \left\{(p+q+m-n) \phi-\nu \pi-\frac{\pi}{2}\right\} \\
& +\cos \left\{(q+m+n-p) \phi-\nu \pi-\frac{\pi}{2}\right\} \\
& +\cos \left\{(m+n+p-q) \phi-\nu \pi-\frac{\pi}{2}\right\} \\
& +\cos (m+n-p-q) \phi \\
& +\cos (m+p-n-q) \phi+\cos (m+q-n-p) \phi
\end{aligned}
$$

we readily see that, for the special case when $\sigma=a$, namely, a rigid quadrilateral with area zero, the integral (3.1) is properly divergent for $\nu<0$; however, for $\nu \geqq 0$, it converges (and $=0$ ). We further note that for the case when $\sigma=a+d=b+c$, (3.1) diverges for $\nu \leqq 0$.

For the subsequent considerations we shall assume that a quadrilateral is constructible, and notice that the construction may be arranged in several ways. Let us consider one of the arrangements, say, the quadrilateral is formed by sides $a, b, c, d$ which are connected with one another in the related order. One of the diagonals, which is denoted by $R$, divides the quadrilateral into two triangles 
$\triangle a b R$ and $\Delta c d R$. Since the diagonal $R$ is of variable length, we let $R=c+d-2 t$ (or $R=a+b-2 u$ ) where $t$ (or $u$ ) is a variable. We further denote the area of the two triangles by $\Delta_{a b R}$ and $\Delta_{c d R}$ respectively.

For sake of definiteness, suppose $c-d \leqq a-b$; when the equality sign is used we remember that $\nu>0$. Replacing $b$ by $c$, and $c$ by $d$ in (2.7), multiplying both sides of the modified equation by $x^{1-\nu} J_{\nu}(a x) J_{\nu}(b x)$ and then integrating with respect to $x$ from 0 to $\infty$, we obtain

$$
\begin{aligned}
S(\nu ; a, b, c, d)= & \int_{0}^{\infty} x^{1-\nu} J_{\nu}(a x) J_{\nu}(b x) \\
& \cdot\left\{\int_{c-d}^{c+d} \frac{2^{\nu-1} J_{\nu}(R x) \Delta_{c d R}^{2 \nu-1}}{\Gamma(1 / 2) \Gamma(\nu+1 / 2)(R c d)^{\nu}} R d R\right\} d x \\
= & \frac{2^{\nu-1}}{\Gamma(1 / 2) \Gamma(\nu+1 / 2)(c d)^{\nu}} \int_{c-d}^{c+d} \frac{\Delta_{c d R}^{2 \nu-1}}{R^{\nu-1}} \\
& \cdot\left\{\int_{0}^{\infty} J_{\nu}(a x) J_{\nu}(b x) J_{\nu}(R x) x^{1-\nu} d x\right\} d R .
\end{aligned}
$$

Here the interchange of the above integrations is permissible for $\nu>-1 / 2$, since the improper integral $S(\nu ; a, b, R)$ is uniformly convergent in general with respect to $R$ in the interval $c-d$ to $c+d$; and

$$
\int_{c-d}^{R} \frac{2^{\nu-1} J_{\nu}(R x) \Delta_{c d R}^{2 \nu-1}}{\Gamma(1 / 2) \Gamma(\nu+1 / 2)(R c d)^{\nu}} R d R
$$

is a continuous function of $R$ for the range $c-d \leqq R \leqq c+d[4$, p. 473]. Write $R=c+d-2 t$ and by Sonine's formula (1.2) we may rewrite (3.5) as follows:

$$
\begin{aligned}
& S(\nu ; a, b, c, d) \\
& \quad=\frac{2^{2 \nu-1}}{\pi\{\Gamma(\nu+1 / 2)\}^{2}(a b c d)^{\nu}} \int_{0}^{\sigma-a}\left(\frac{c+d-2 t}{\Delta_{a b R} \Delta_{c d R}}\right)^{1-2 \nu} d t,
\end{aligned}
$$

which we shall call the generalized Sonine's integral formula.

4. Generalized Dougall's expansion formula. Similar to the related line of reasoning, we use instead of (2.7) the known result $[5$, p. 378]

$$
\begin{aligned}
\int_{|\beta-\gamma|}^{B} P_{n}^{(\lambda)}(\cos \alpha) & (\sin \alpha \sin \beta \sin \gamma)^{1-2 \lambda} E^{\lambda-1} \sin ^{2 \lambda} \alpha d \alpha \\
= & 2\{\Gamma(\lambda)\}^{2} \frac{\Gamma(n+1)}{\Gamma(n+2 \lambda)} P_{n}^{(\lambda)}(\cos \beta) P_{n}^{(\lambda)}(\cos \gamma)
\end{aligned}
$$


where $P_{n}^{(\lambda)}(\cos \theta)$ denotes ultra-spherical polynomial. In (4.1) we note that $\beta$ and $\gamma$ are two fixed sides of a spherical triangle: $0<\beta<\pi$, $0<\gamma<\pi ; \alpha$ is the third side; that

$$
E=\sin \frac{\alpha+\beta+\gamma}{2} \sin \frac{\beta+\gamma-\alpha}{2} \sin \frac{\gamma+\alpha-\beta}{2} \sin \frac{\alpha+\beta-\gamma}{2}
$$

and that $B=\beta+\gamma$ if $\beta+\gamma \leqq \pi$; or $B=2 \pi-\beta-\gamma$, if $\beta+\gamma>\pi$.

Suppose $\alpha \geqq \beta \geqq \gamma \geqq \delta$, and for sake of definiteness let $\gamma-\delta \leqq \alpha-\beta$. For the latter relation when the equality sign is used, we consider only $\lambda>1 / 2$. Now replacing $\beta, \gamma, \alpha, E, B$ in (4.1) by $\gamma, \delta, \rho, E_{\gamma \delta \rho}, B^{\prime}$ respectively; multiplying both sides of the modified equation by

$$
(n+\lambda)\left\{\frac{\Gamma(n+1)}{\Gamma(n+2 \lambda)}\right\}^{2} P_{n}^{(\lambda)}(\cos \alpha) P_{n}^{(\lambda)}(\cos \beta),
$$

and then summing with respect to $n$ from 0 to $\infty$, we finally obtain

$$
\begin{aligned}
D_{2}(\lambda ; \alpha, \beta, \gamma, \delta) & \begin{aligned}
\sum_{n=0}^{\infty}(n+\lambda)\left\{\frac{\Gamma(n+1)}{\Gamma(n+2 \lambda)}\right\}^{3} \\
\quad \cdot P_{n}^{(\lambda)}(\cos \alpha) P_{n}^{(\lambda)}(\cos \beta) P_{n}^{(\lambda)}(\cos \gamma) P_{n}^{(\lambda)}(\cos \delta)
\end{aligned} \\
= & \frac{1}{2}\{\Gamma(\lambda)\}^{-2} \sum_{n=0}^{\infty}(n+\lambda)\left\{\frac{\Gamma(n+1)}{\Gamma(n+2 \lambda)}\right\}^{2} P_{n}^{(\lambda)}(\cos \alpha) P_{n}^{(\lambda)}(\cos \beta) \\
& \cdot \int_{\gamma-\delta}^{B^{\prime}}(\sin \rho \sin \gamma \sin \delta)^{1-2 \lambda} E_{\gamma \delta \rho}^{\lambda-1} P_{n}^{(\lambda)}(\cos \rho) \sin ^{2 \lambda} \rho d \rho \\
= & \frac{1}{2}\{\Gamma(\lambda)\}^{-2} \int_{\gamma-\delta}^{B^{\prime}}(\sin \rho \sin \gamma \sin \delta)^{1-2 \lambda} E_{\gamma \delta \rho}^{\lambda-1} \sin ^{2 \lambda} \rho \\
& \cdot\left\{\sum_{n=0}^{\infty}(n+\lambda)\left\{\frac{\Gamma(n+1)}{\Gamma(n+2 \lambda)}\right\}^{2}\right. \\
& \left.\cdot P_{n}^{(\lambda)}(\cos \alpha) P_{n}^{(\lambda)}(\cos \beta) P_{n}^{(\lambda)}(\cos \rho)\right\} d \rho .
\end{aligned}
$$

Here the interchange of the infinite series and the integration is permissible for $\lambda>0$, since the infinite series is uniformly convergent with respect to $\rho$ in the interval between $\gamma-\delta$ and $B^{\prime}$; and the integral

$$
\int_{\gamma \rightarrow \delta}^{B^{\prime}} E_{\gamma \delta \rho}^{\lambda-1} \sin \rho d \rho
$$


is absolutely convergent [2, p. 495]. By the result of Dougall's expansion formula we may rewrite (4.3) as follows:

$$
\begin{aligned}
D_{2}(\lambda ; \alpha, \beta, \gamma, \delta)= & 2^{-1-2 \lambda} \pi\{\Gamma(\lambda)\}^{-6}\{\sin \alpha \sin \beta \sin \gamma \sin \delta\}^{1-2 \lambda} \\
& \cdot \int_{\gamma-\delta}^{B^{\prime}}\left\{\frac{E_{\alpha \beta \rho} E_{\gamma \delta \rho}}{\sin ^{2} \rho}\right\}^{\lambda-1} d \rho,
\end{aligned}
$$

which we shall call the generalized Dougall's expansion formula. We shall remark that by means of a limiting process $[5$, p. 380] the generalized Sonine's integral formula (3.6) may also be derived from (4.4).

5. Generalized Dougall's integral formula. Bailey $[1$, p. 284] has attempted to generalize Busbridge's formula [3, p. 95], which involves products of Hermite polynomials, by evaluating the integral

$$
\begin{aligned}
D_{1}(\lambda ; m, n, p, q) \\
\quad=\int_{-1}^{1}\left(1-x^{2}\right)^{\lambda-1 / 2} P_{m}^{(\lambda)}(x) P_{n}^{(\lambda)}(x) P_{p}^{(\lambda)}(x) P_{q}^{(\lambda)}(x) d x .
\end{aligned}
$$

He did not give an explicit, elementary expression for this generalized integral, but remarked that the result is complicated and involves a well-poised series of the type ${ }_{11} F_{10}$ with unit argument.

By the known result of Dougall's integral formula [5, p. 374] we immediately have

$$
P_{p}^{(\lambda)}(x) P_{q}^{(\lambda)}(x)=\sum_{r=0}^{q} \pi_{r}^{(\lambda ; p, q)} P_{p+q-2 r}^{(\lambda)}(x),
$$

where

$$
\begin{aligned}
\pi_{r}^{(\lambda ; p, q)}= & \frac{p+q-2 r+\lambda}{p+q-r+\lambda} \frac{\Gamma(p+q-2 r+1)}{\Gamma(p+q-r+1)} \\
& \frac{\Gamma(p+q-r+2 \lambda)}{\Gamma(p+q-2 r+2 \lambda)} \\
& \frac{\left(\begin{array}{c}
p-r+\lambda-1 \\
p-r
\end{array}\right)\left(\begin{array}{c}
q-r+\lambda-1 \\
q-r
\end{array}\right)\left(\begin{array}{c}
r+\lambda-1 \\
r
\end{array}\right)}{\left(\begin{array}{c}
p+q-r+\lambda-1 \\
p+q-r
\end{array}\right)} .
\end{aligned}
$$

Now by an analogous method, as employed by Bailey in dealing with Hermite polynomials, we may obtain an explicit expression for the value of the integral (5.1), namely: 


$$
\begin{aligned}
& D_{1}(\lambda ; m, n, p, q) \\
& =\frac{2^{1-2 \lambda}}{\{\Gamma(\lambda)\}^{2}} \sum_{r=0}^{\min (q, k-m)} \frac{\pi}{k-r+\lambda} \frac{\Gamma(k-r+2 \lambda)}{\Gamma(k-r+1)} \frac{p+q-2 r+\lambda}{p+q-r+\lambda} \\
& \frac{\Gamma(p+q-2 r+1)}{\Gamma(p+q-r+1)} \frac{\Gamma(p+q-r+2 \lambda)}{\Gamma(p+q-2 r+2 \lambda)} \\
& \frac{\left(\begin{array}{c}
p-r+\lambda-1 \\
p-r
\end{array}\right)\left(\begin{array}{c}
q-r+\lambda-1 \\
q-r
\end{array}\right)\left(\begin{array}{c}
r+\lambda-1 \\
r
\end{array}\right)}{\left(\begin{array}{c}
p+q-r+\lambda-1 \\
p+q-r
\end{array}\right)} \\
& \left(\begin{array}{c}
k-m-r+\lambda-1 \\
k-m-r
\end{array}\right)\left(\begin{array}{c}
k-n-r+\lambda-1 \\
k-n-r
\end{array}\right)\left(\begin{array}{c}
k-p-q+r+\lambda-1 \\
k-p-q+r
\end{array}\right) \\
& \left(\begin{array}{c}
k-r+\lambda-1 \\
k-r
\end{array}\right) \\
& =\frac{2^{1-2 \lambda}}{\{\Gamma(\lambda)\}^{2}} \sum_{r=0}^{\min } F_{r}^{(q, k-m)}(\lambda ; m, n, p, q),
\end{aligned}
$$

where we let $2 k=m+n+p+q$ and $m \geqq n \geqq p \geqq q, k$ being an integer not less than $m$; otherwise the integral is zero. For $\lambda=1 / 2$, the explicit expression for the integral involving product of Legendre polynomials was given by Shabde [6, pp. 31-32]. We may further remark that by means of a limiting process [5, p. 379], (5.4) will contain the same generalized Sonine's integral formula (3.6) as a special case.

We finally note that Bailey $[1$, p. 282] also remarked that for any four positive numbers, say $m \geqq n \geqq p \geqq q$, there are several ways of obtaining the value of the integral (5.1). It is easy to show, for example, with $n$ and $q$ interchanged in the related order and following the analogous argument for the evaluation, that we have

$$
\begin{aligned}
D_{1}(\lambda ; m, n, p, q) & =D_{1}(\lambda ; m, q, p, n) \\
& =\frac{2^{1-2 \lambda}}{\{\Gamma(\lambda)\}^{2}} \sum_{r=\max (0, k-m-q)}^{k-m} F_{r}(\lambda ; m, q, p, n) .
\end{aligned}
$$

Making use of a limiting process and particularly for the case when $\nu=0$, we have, if $a_{1} \geqq a_{2} \geqq a_{3} \geqq a_{4}>0$, and $\omega=a_{2}+a_{3}-2 x$,

$$
\begin{aligned}
S\left(0, a_{1}, a_{2}, a_{3}, a_{4}\right) & =\int_{0}^{\infty} \prod_{n=1}^{4} J_{0}\left(a_{n} t\right) t d t \\
& =\frac{1}{2 \pi^{2}} \int_{\max \left(0, \sigma-a_{1}-a_{4}\right)}^{\sigma-a_{1}} \frac{a_{2}+a_{3}-2 x}{\Delta_{a_{1} a_{4} \omega} \Delta_{a_{2} a_{3} \omega}} d x .
\end{aligned}
$$


We obtain

$$
\begin{aligned}
& S\left(0 ; a_{1}, a_{2}, a_{3}, a_{4}\right)=\frac{4}{\pi^{2}} \int_{a_{1}-a_{4}}^{\min \left(a_{2}+a_{3}, a_{1}+a_{4}\right)}\left[\left\{\left(a_{1}+a_{4}\right)^{2}-\omega^{2}\right\}\right. \\
& \left.\cdot\left\{\left(a_{1}-a_{4}\right)^{2}-\omega^{2}\right\}\left\{\left(a_{2}+a_{3}\right)^{2}-\omega^{2}\right\}\left\{\left(a_{2}-a_{3}\right)^{2}-\omega^{2}\right\}\right]^{-1 / 2} \omega d \omega,
\end{aligned}
$$

which is the well known Nicholson's result [8, p. 414].

\section{REFERENCES}

1. W. N. Bailey, On Hermite polynomials and associated Legendre functions, J. London Math. Soc. vol. 14 (1939) pp. 281-286.

2. T. J. I'a. Bromwich, An introduction to the theory of infinite series, Macmillan, 1926.

3. I. W. Busbridge, The evaluation of certain integrals involving products of Hermite polynomials, J. London Math. Soc. vol. 14 (1939) pp. 93-97.

4. G. A. Gibson, An elementary treatise on the calculus, Macmillan, 1922.

5. Hsien-yü Hsü, Certain integrals and infinite series involving ultraspherical poly. nomials and Bessel's functions, Duke Math. J. vol. 4 (1938) pp. 374-383.

6. N. G. Shabde, On a definite integral involving Legendre functions, Proceedings of the Benares Mathematical Society N.S. vol. 5 (1943) pp. 31-32.

7. G. Szegö, Über gewisse Potenzreihen mit lauter positiven Koeffizienten, Math. Zeit. vol. 37 (1932) pp. 674-688.

8. G. N. Watson, A treatise on the theory of Bessel functions, Cambridge, 1922.

YENCHING UNIVERSITY 